\title{
3D IMAGE RECONSTRUCTION OF OVARIAN TUMOR IN THE ULTRASONIC IMAGES
}

\author{
C. F. JIANG \\ Dept. of Biomedical Engineering, I-Shou University \\ Kaohsiung, Taiwan
}

\begin{abstract}
The prevalence of ovarian tumor malignancy can be monitored by the degree of irregularity in
the ovarian contour and by the septal structure inside the tumor observed in ultrasonic images.
However the $2 D$ ultrasonic images can not integrate $3 D$ information form the ovarian tumor. In this
paper, we present an algorithm that can render the $3 D$ image of an ovarian tumor by reconstructing
the $2 D$ ultrasonic images into a $3 D$ data set. This is based on sequentially boundary detection in a
series of $2 D$ images to form a $3 D$ tumor contour. This contour is then used as a barrier to remove
the data containing the other tissue adhering to the tumor surface. The final $3 D$ image rendered by
the isolated data provides a clear view of both the surface and inner structure of the ovarian tumor.
Biomed Eng Appl Basis Comm, 2001 (April); 13: $93-98$.
Keyword: Ovarian tumor, Ultrasonic image, 3D reconstruction, Boundary detection
\end{abstract}
\section{INTRODUCTION}

Ovarian cancer is one of the most mortal female cancers found in civilized countries. Among the various detection methods for ovarian cancer, the ultrasonic technique is the most common due to its noninvasive properties and its high degree of sensitivity and specificity. In addition, the degree of irregularity of the ovarian contour and the septal structure inside the tumor observed in ultrasonic images are the most important indices indicating the prevalence of the malignancy in the ovarian tumor $[1,2]$. It may, however be inappropriate to determine these indices only by $2 \mathrm{D}$ ultrasonic image observation. It is believed that global inspection of a 3D ovarian tumor image can improve the objectivity and accuracy in the detection of ovarian cancer[3]. Hence, this motivates us to develop an algorithm to reveal a clear image of 3D structural of the ovarian tumor.

Received: Jan. 16, 2001; accepted: March 5, 2001.

Correspondence: C. F. Jiang, Ph.D.

Dept. of Biomedical Engineering, I-Shou University Ta-Hsu Hsiang, Kaohsiung, Taiwan
A clear view of a 3D entity in a medical image usually can not be achieved by simply applying the typical 3D image rendering techniques because of interference by the surrounding tissue. Therefore, a method to detect the 3D contour of the tumor must be developed first to produce a barrier to remove the nonovarian tissue to render only the ovarian tumor.

The rest of this paper is organized as follows: The details of the algorithm are given consecutively in section 2, followed by the results of each procedure with discussion in section 3 . Section 4 makes a brief conclusion for this study.

\section{METHOD}

A series of 2D image data were compiled into a volume data were obtained from VOLUSON 530D by Kretz Technik. This system contains a probe with a rotating phase-array transducer that can scan multipleplan images for each complete acquisition.

2D images were firstly classified into 3 classes. Based on the classified image, the tumor boundaries in a series of 2-D ultrasonic images were detected to form a shell in the volume data. This shell was used to select the data for the 3D image rendering. Both sur- 
face and volume rendering techniques were adopted to yield the 3D images to provide both interior and exterior view of the ovarian tumor

\subsection{Image Classification via Feature Extrac- tion}

According to Law's approach [6], a set of feature masks representing different operations can be derived by convolution process. This starts from the convolution with three basic vectors with lengths of 3 , $\mathrm{L} 3=(1,2,1), \quad \mathrm{E} 3=(-1,0,-1)$, and $\mathrm{S} 3 z(-1,2,-1)$. These three vectors represent the one-dimensional operations of center-weighted local averaging, symmetrical first differencing for edge detection, and second differencing for spot detection. If these vectors are convolved with themselves or with one another, 9 vectors with length of 5 can be obtained. However, 3 pairs of these vectors are additive inverse to one another and E3*E3 is similar to $\mathrm{L3}^{*} \mathrm{~S} 3$. Excluding these vectors 5 vectors representing different operations remain. The remaining vectors include a local averaging filter $\mathrm{L} 5=(1,4,6,4,1)$, edge detector $\mathrm{E} 5=(-1,-2,0,2,1)$, spot detector $S 5=(-1,0,2,0,-1)$, ripple detector $R 5=(1$, $-4,6,-4,1)$ and wave detector W5 $2(-1,2,0,-2,1)$.

If the column vectors, with a length of 5 , are multiplied by row vectors with the same length, 25 Laws' $5 \times 5$ masks are obtained. Theoretically, these 25 masks can extract 25 textural patterns by convolving these masks with an image. For example, the $\mathrm{L}^{\mathrm{l}}{ }^{*} \mathrm{E} 5$ mask can detect the vertical edges existing in the image and average the differences across the vertical direction. The $E 5^{t^{*}}$ L5 mask can detect the horizontal edges and average the differences across the same direction. In our previous study $[4,5]$, the derivation of the Laws' feature masks is re-evaluated and the results suggests a clear pattern from the ultrasonic image of the ovary can only be extracted by using five masks; i.e., $\mathrm{L}^{\prime *} \mathrm{~L}^{\mathrm{t}}, \mathrm{L}^{\mathrm{i}}{ }^{*} \mathrm{E} 5, \mathrm{~S}^{\prime} * \mathrm{E} 5, \mathrm{E} 5^{\prime *} \mathrm{~L} 5$, and $\mathrm{E5}^{\mathrm{t} *} \mathrm{E5}$. (figure 1). These masks are then used to extract the features on which the pixel classification process is based.

The basic paradigm used in pattern classification is to make $n$ measurements on the entity to be classified and then consider the result as a point in an $n$ dimensional feature space. A 5-dimensional feature space is used for the $\mathrm{K}$-means clustering process, due to the selection of five features for each pixel in the image.
The $\mathrm{K}$-means clustering algorithm is applied to classify each pixel in the image into 3 regions, nonovarian, normal and abnormal ovarian tissue. The clustering process is iterative. First, three seed points were determined by selecting three feature vectors, which are the centers of three regions equally divided in the feature space. The current seed points are used to classify all of the pixels according to the minimum Euclidean distances between their feature vectors and the seed points. The seed points are then recalculated as the means of all the pixels in the same classes. This process is repeated until all of the means shift less than a fixed amount between iterations.

The classified results are assigned into three graylevels with intensity values of 0,100 , and 150 , in terms of black, dark gray and light gray color in the image to represent fluid, ovarian, and other connective tissue, respectively.

\subsection{Finding the 3D Tumor Contour by Edge Detection}

Based on the characteristics of the classified results, the tumor contour can be found by detecting all of the boundaries in the sequence of $2 \mathrm{D}$ images. $\mathrm{A}$ search algorithm was developed to avoid fragmented edges due to interference from septal tissue, which is also classified into a gray color. The 3D contour of the ovarian tumor is detected using the following steps:

\section{Step 1: Define search lines}

All of the pixels with the maximal intensitygradient on the image are selected and the mean of these point positions is calculated as the center of the tumor. From the center we can plot $360 / \mathrm{N}$ radiate search lines separated by $\mathrm{N}$ degrees.

\section{Step 2: Find the Candidate Edge Points (Pc)}

$$
P_{c}=\left\{P_{i} \mid \operatorname{gray}\left(P_{i}\right) \text {-gray }\left(P_{j-1}\right)=+50, P_{i}:\right. \text { all points }
$$
in the search line, $\mathrm{i}: 0,1,2, \cdots, \mathrm{n} ; \mathrm{P}_{0}$ locates at the center $\}$, where gray $\left(P_{i}\right)$ denotes the gray-value of $P_{i}$

\section{Step 3: Select the Real Edge Points}

Select the real edge point $(P)$ from the candidate points $\left(P_{c}\right)$. This is accomplished by approximating the shape of the ovary with an initial contour, which is an ellipse for the first slice or the boundary of the previous slice for the remaining slices. A search is then made for the real edge point with the minimum dis-

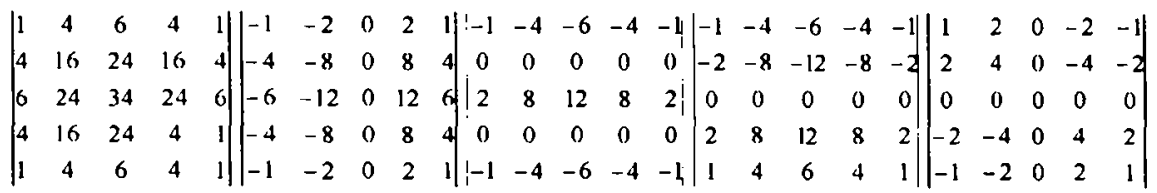

Fig. 1 Five Laws' feature masks (from left to right, top to bottom: $\mathrm{LS}^{\mathrm{t}^{*}} \mathrm{LS}^{\mathrm{t}}, \mathrm{LS}^{\mathrm{t*}} \mathrm{E}$, $\mathrm{S5}^{\mathrm{t}^{*}} \mathrm{E5}, \mathrm{E} 5^{\mathrm{t}^{*}} \mathrm{L5}$, and E5 ${ }^{1 *}$ E5) 


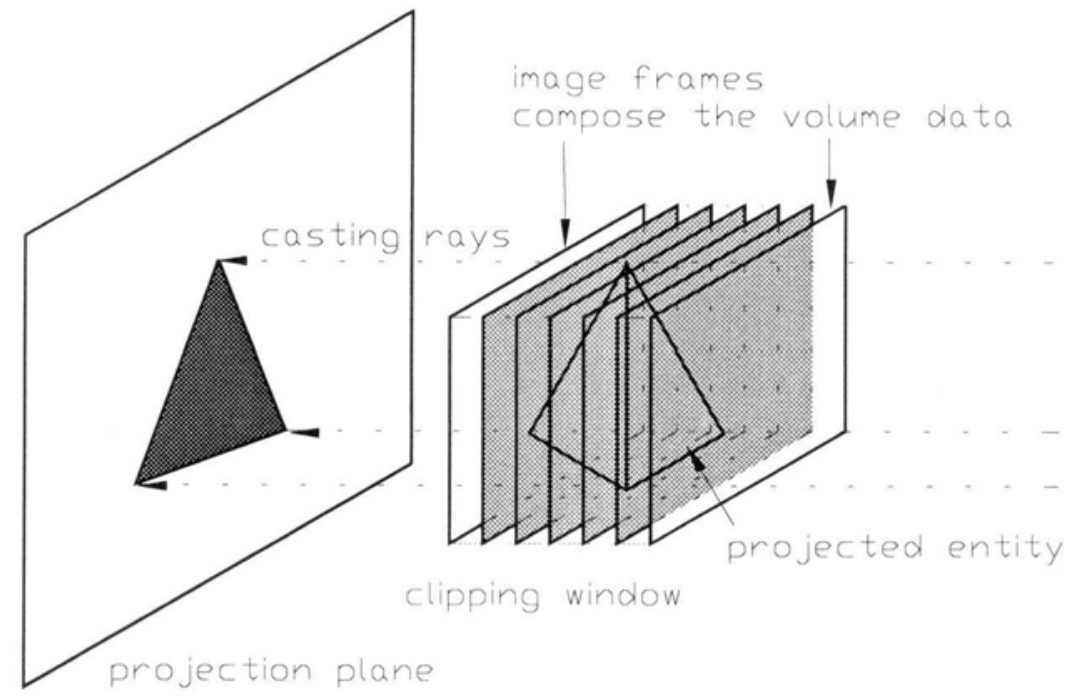

Fig. 2 the theory of MIP. The clipping window (in gray color) is used to select the image frames which cover the portion of the entity for observation
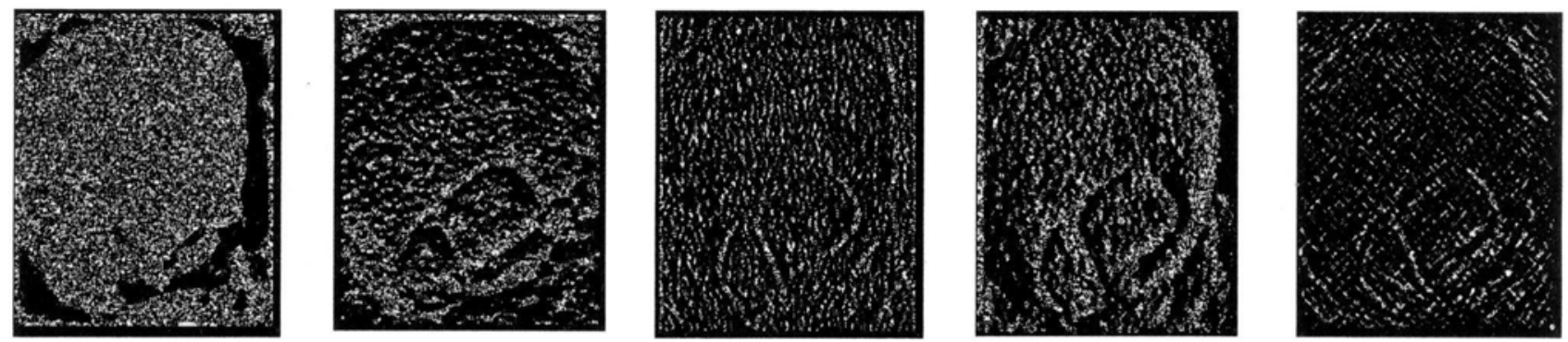

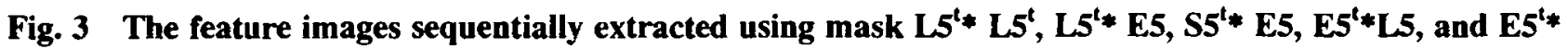
E5

tance to the initial contour $(C)$.

$P=\left\{P_{c}|\min | P_{c} \cdot C \mid\right\}$

Step 4: Repeat Step3 on every search line.

Step 5: Interpolate all edge points using a cubic spline [14] to obtain the continuos ovarian edge.

\subsection{D Image Reconstruction}

The detected contour was used as a shell inside which the volume data were preserved for the volume rendering process. This procedure tends to isolate the tumor and provide a clear view of its inner structure without concealment by non-ovarian tissue during the 3D image rendering. In addition, the binary volume data, which was obtained by assigning 1 to the voxel containing the shell and 0 to the rest, were used for the surface rendering techniques to yield the 3D image of the contour surface, which provides an inspection of the irregularity of the tumor surface.

\subsubsection{Inspection of the Inner Structure by MIP}

The septa structure inside the tumor appears as brighter than its surrounding liquid in the $2 \mathrm{D}$ images. This encourage us to adapt MIP [10] to reveal the 3D septa geometry. A MIP is constructed by casting parallel rays through the preserved volume data. As each ray passes through the data, the brightest voxel in each ray is projected into a viewing plane, which is perpendicular to the rays, as shown in figure 2 . As a result of the transformation from 3D data to $2 \mathrm{D}$, depth information is lost. This problem can be solved by continuously projecting the data at several different angles, which requires applying geometric transformations to the voxels-(1) to translate the data set to make it centralized at the origin of the view space, (2) rotate about the $y$ axis, and (3) translate it back to the original location. However, the transformation invariably converts the integer-valued coordinates into real-valued coordinates. In other words, as the volume data rotates, the casting rays may intersect the volume data at the points with fractional coordinates; i.e., no intensity value with these points, as a consequence, the projection plane will contain some holes. 

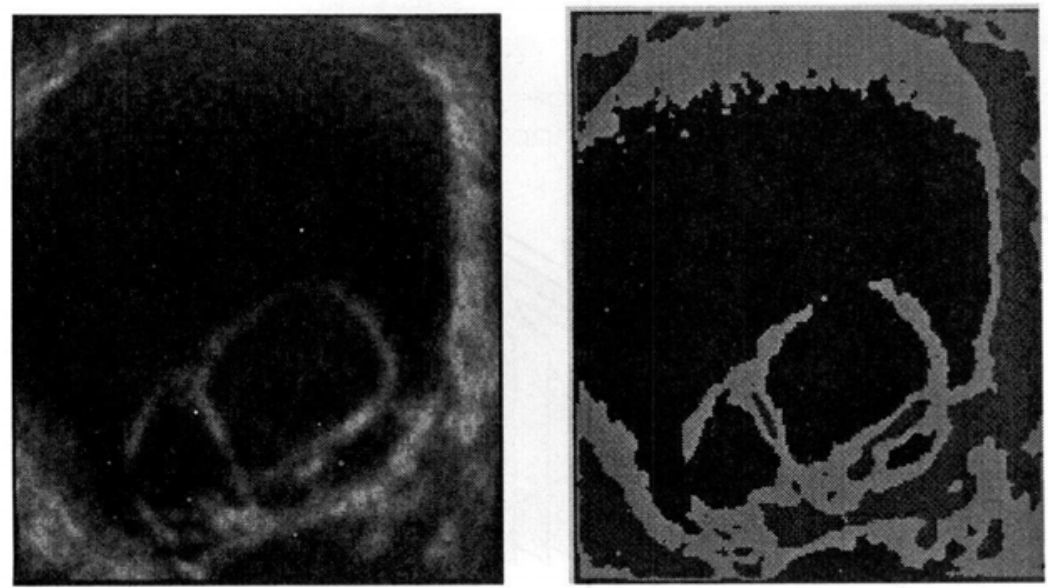

Fig. 4 The classification result (right) of the original image (left)
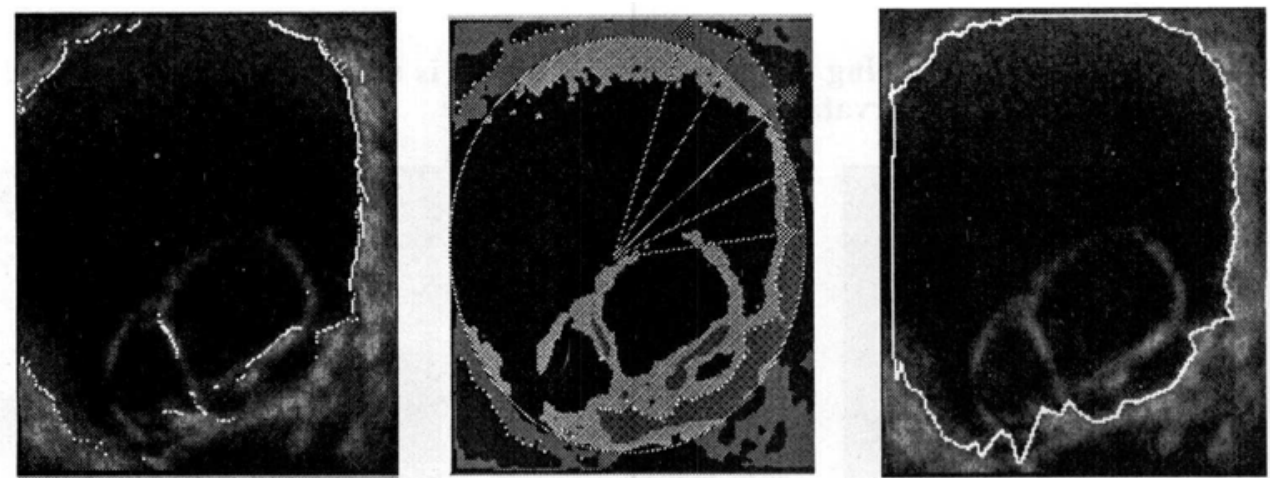

Fig. 5 Left: the detected edge (white dots) using intensity gradient only; Middle: edge detection algorithm refined using the initial model; Right: the detected boundary using the refined model

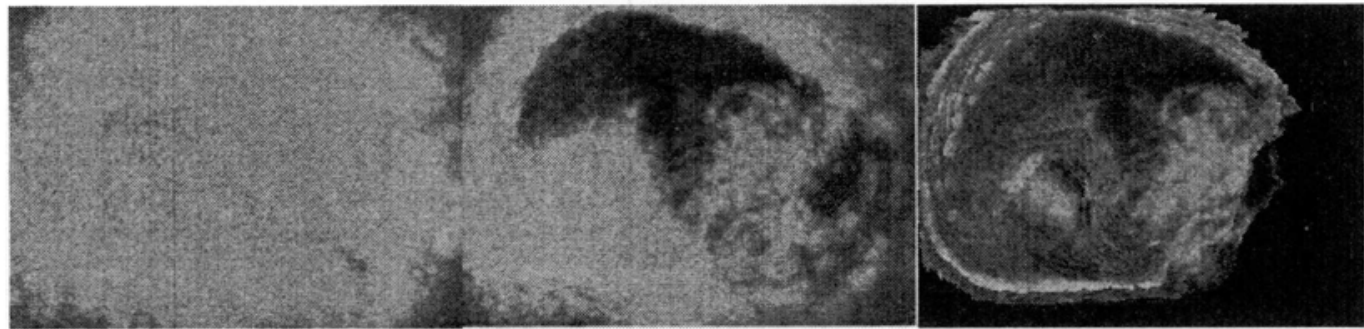

Fig. $63 \mathrm{D}$ images rendered using MIP applied to (left) raw data (middle) clipping data (right) selected data using the 3D contour. (details refer to the text)

Some interpolation methods have been proposed to calculate the values of those points according to their neighbors [11-13]. The derivation process in these methods may be very precise but rather time consuming. Therefore we developed a fast algorithm to find values to fill these holes: simply reverse the geometric transformation from step (3) to (1) to trace back the original voxel values of these points before rotation

\subsubsection{Inspection of the 3D Surface by Surface Rendering}

The original volume data is first converted into binary data by assigning 1 to the voxels containing the tumor surface $\left(\hat{i}_{1}\right)$ and 0 to the remaining voxels $\left(i_{0}\right)$. The purpose of this procedure is to select the contour only in the volume data for projection. We assumed orthographic projection, no perspective transformation in viewing, and just as in MIP, a parallel ray light source situated such that the viewing direction is parallel to the light rays and to the image slices. The $i_{1} s$ were then projected at several different angles to the viewing plane using the Z-buffer technique [14] to re- 


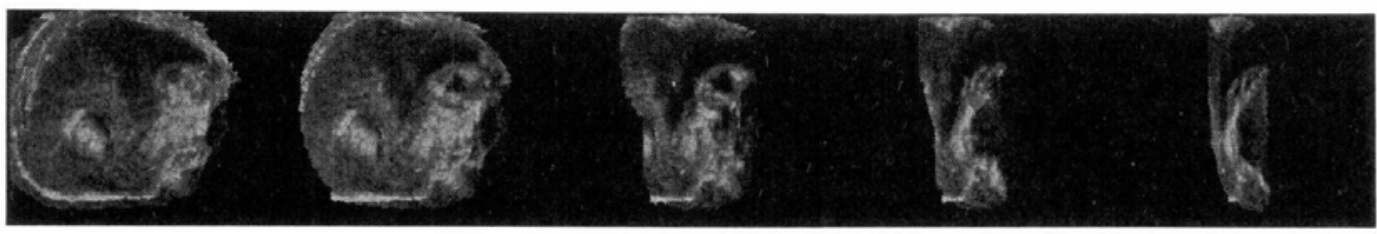

$\begin{array}{lllll}0^{\circ} & 18^{\circ} & 36^{\circ} & 54^{\circ} & 72^{\circ}\end{array}$

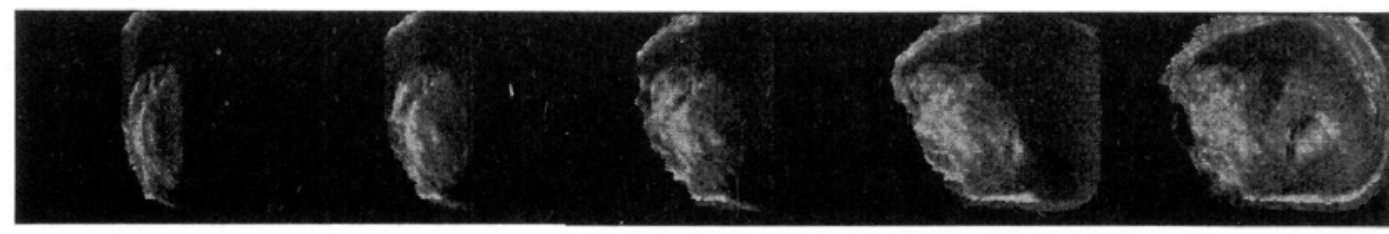

$90^{\circ} \quad 108^{\circ} \quad 126^{\circ} \quad 144^{\circ} \quad 162^{\circ}$

Fig. 7 MIP images as the data rotates an arc by every $18^{\circ}$.

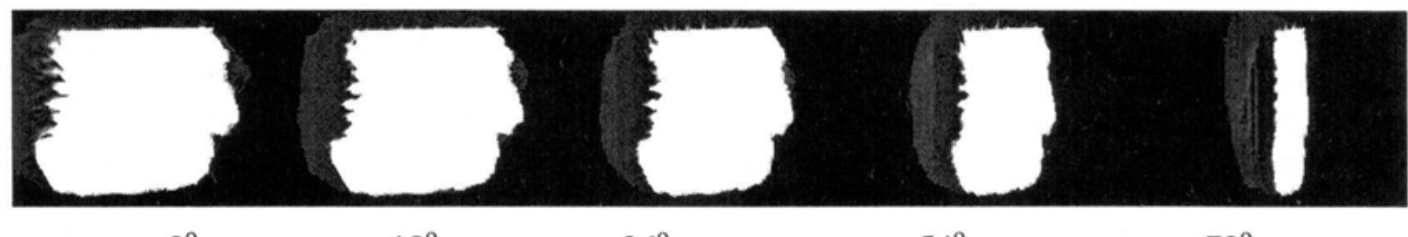

$\begin{array}{lllll}0^{\circ} & 18^{\circ} & 36^{\circ} & 54^{\circ} & 72^{\circ}\end{array}$

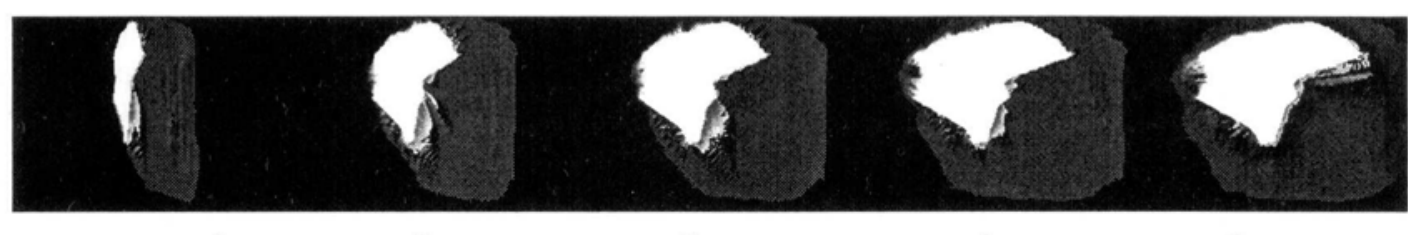

$\begin{array}{lllll}90^{\circ} & 108^{\circ} & 126^{\circ} & 144^{\circ} & 162^{\circ}\end{array}$

Fig. $83 \mathrm{D}$ images of the tumor contour rotating an arc by every $18^{\circ}$.

move the hidden surfaces. For every pixel $p$ in the viewing plane, the intensity value is equal to its corresponding voxel $c$ in the original data set. To obtain a more realistic look for the contour in the viewing plane, we used the Phone shading model to calculate the final intensity for each $\boldsymbol{p}$. The original model, as in equation (1), states that the intensity of $p$ is the sum of the diffusion reflection $f_{d}(n, l)$, specular reflection $f_{s}(n, l)$, and background ambient light $A$, and inversely proportional to the distance from $c$ to the viewing plane. Herein we set $\boldsymbol{k}_{\boldsymbol{d}}$ to 0 , since the rotation of the contour make no difference for diffuse reflection.

$$
I(p)=1 / z(c)\left(k_{d} f_{d}(n, l)+k_{s} f_{s}(n, l)\right)+A
$$

where $\mathrm{kd} / \mathrm{ks}$ represents a weight that determines the diffuse / specular component, respectively. $\mathbf{n}$ is the unit normal of surface; 1 is a unit vector in the direction of the light rays.

\section{RESULTS AND DISCUSSION}

\subsection{Feature Extraction and Edge Detection}

Five clear feature images (figure 3) were obtained by convolving the original image with 5 feature masks; i.e., $\mathrm{L}^{\mathrm{t}} * \mathrm{~L} 5, \mathrm{E} 5^{\mathrm{t} * \mathrm{E} 5}, \mathrm{E} 5^{\mathrm{t}} \mathrm{L} 5, \mathrm{~L} 5^{\mathrm{t}} \mathrm{E} 5$, and $\mathrm{S} 5^{\mathrm{t}} \mathrm{L} 5$. The classified image based on these features is shown in figure 4. In essence, the selection of appropriate features should be the most crucial part of any texturebased segmentation problem, because an improper choice of the features will lead to incorrect segmentation. We have been tried the other feature sets, $\mathrm{E} 5^{\mathrm{t}} \mathrm{S} 5, \mathrm{~L}^{\mathrm{t}} * \mathrm{~S} 5, \mathrm{R} 5^{\mathrm{t}} \mathrm{R} 5$ and $\mathrm{E} 5^{\mathrm{t}} \mathrm{L} 5$, which were successfully applied to other medical image segmentation problems $[7,8]$. However, our segmentation task with these features was unsuccessful. The cause of this failure can be explored in the clustering process described in the method section. While calculating the Euclidean distance, every element in the feature 
vector has the same weight. Therefore, if the features are not properly selected, the effect of good features will be averaged with bad features and reduce the accuracy of the classification result. In this study, we found that the new feature set led to a segmentation result of ultrasonic image of ovarian tumor close corresponding to human vision.

Once the image has been classified, the edge becomes more distinguished. Inspecting the classified image in figure 4 , the edge exits roughly between the dark gray area outward and light gray area inward. Furthermore, some tissue adhering to the tumor boundary is also revealed. If this rule is applied only to detect the edge, some noise and the septa structure will cause a false edge as indicated in the left image in figure 5. This problem can be solved by our initial boundary approach (middle image in figure 5 ), which can reveal an integrated boundary (right image in figure 5 ).

\subsection{D Image Rendering}

Figure 6 shows the results comparison of MIP applied to the raw data (left), to the selected data using a clipping window, and to the preserved data inside the tumor contour. It can been seen that the other tissue around the tumor strongly affects the MIP results. Even clipping the volume data still can not delete the effect of the adhering tissue. Only using the $3 \mathrm{D}$ contour can totally remove the adhering tissue and yield an integrated view of interior structure of the tumor.

Figure 7 shows the final MIP images as the data rotates from $0^{\circ}$ to $180^{\circ}$ every $18^{\circ}$. The decreasing size of the tumor from $36^{\circ}$ to $126^{\circ}$ is due to the shorter volume depth in comparison with its width and length. This is caused by clipping the unnecessary slices beside the tumor from the volume data (as in figure 2).

Figure 8 shows the images of the 3D tumor contour rotating from $0^{\circ}$ to $180^{\circ}$ every $18^{\circ}$. The reason for the decreasing in the size of the tumor from $36^{\circ}$ to $126^{\circ}$ is the same as that for MIP. The white parts in the images indicate the clipping surfaces. The irregular site can been seen as the contour rotating to $72^{\circ}$ and $90^{\circ}$.

\section{CONCLUSION}

The contribution of this study can be concluded as: (1) A new set of Laws'-masks was proposed to clearly reveal the ovarian features in an ultrasonic image. This result also suggests that different kind of images should use different set of Laws' feature masks. (2) An initial-contour-based edge detection technique was developed to overcome the disadvantage of the intensity-gradient-based edge detection technique, i.e., the edge between different tissues is blurred and usually smeared by speckle noises. (3) We proposed a method to detect the ovarian contour in the classified image . The volume within the detected contour was used to reconstruct the $3 \mathrm{D}$ image of the ovarian tumor, which provides a clear interior view of both the surface and the inner structure of the ovarian tumor.

\section{REFERENCES}

1. Karlan BY, Platt LD :Ovarian Cancer ScreeningThe Role of Ultrasound in Early detection. Cancer supplement. 1995; 76(10):2011-2015

2. DePriest PD, et. al..: Transvaginal Sonography as a Screening Method for the Detection of Early Ovarian Cancer. Gynecol Oncol. 1997; 65: 408-414

3. Bonilla-Mousoles F, Rafa F, and Osborne NG: Three Dimensional Ultrasound Evaluation of Ovarian Cancer. Gynecol Oncol. 1995; 59(1): 129-135

4. Jiang CF and Chen ML: A Comparative Study of Using Texture Features to Classify Ultrasonic Ovarian Images, Proc. of International Conf. Image and Vision Computing. 1998; pp222-227,

5. Jiang CF and Chen ML: "Segmentation of Ultrasonic Ovarian Images by Texture Features", $20^{\text {th }}$ Annual international Conference of the IEEE Engineering in Medicine and Biology Society, 185, 1998

6. Laws K I: Texture energy measures. Proc. Image Understanding Workshop. 1979; pp. 41-51

7. Richard WD, et al. : Automated texture-based segmentation of ultrasound images of the prostate. Computerized Medical Imaging and Graphics. 1996; 20(3): 131-140

8. Wu CM , and Chen YC: Texture features for classification of ultrasonic liver images. IEEE Trans. Med. Imaging, 1992; 11(2): 141-152

9. Hsiao JY, and Sawchuk AA: Unsupervised textured image segmentation using feature smoothing and probabilistic relaxation techniques. CGIP. 1989; 48: $1-21$

10. Schreiner S, and Galloway RL : A Fast Maximum-Intensity Projection Algorithm for Generation Magnetic Resonance Angiograms. IEEE Trans on Medical Imaging.1993; 12(1): 50-57

11. Keys R G : Cubic Convolution Interpolation for Digital Image Processing. IEEE Trans. on Acoustics Speech and Signal Processing. 1981; 29(6):1153-1160.

12. Raya SP and Udupa JK: Shape-Based Interpolation of Multidimensional Objects. IEEE Trans. Medical Imaging. 1990; 9(1): 32-42

13. Herman GT, Zheng J, and Bucholtz CA: ShapeBased Interpolation. IEEE Computer Graphics \& Applications. 1992; 12(3): 69-79

14. Alan Watt.: "3D Computer Graphics ", $2^{\text {nd }}$, Assison-Wesley publishing company. 1993 